\title{
THE FIRST EIGENVALUE OF THE LAPLACIAN FOR PLANE DOMAINS
}

\author{
CHRISTOPHER B. CROKE ${ }^{1}$
}

\begin{abstract}
We prove an improved lower bound for the first eigenvalue of the Laplacian of a connected plane domain in terms of its inradius and connectivity.
\end{abstract}

In this note we find a lower bound for the first eigenvalue, $\lambda_{1}$, of the Dirichlet problem for the Laplacian of a connected plane domain $D$ in terms of its inradius $\rho$ (the radius of the largest disk contained in $D$ ), and the connectivity $k$ (i.e., the number of boundary components).

Hayman [3] was the first to prove an inequality of this type. He proved $\lambda_{1}(D)>1 / 900 \rho^{2}$ in the case $k=1$ (i.e., $D$ simply connected). Next, Osserman [4] showed that

$$
\begin{aligned}
& \lambda_{1}(D) \geqslant 1 / 4 \rho^{2}, \quad k=1,2, \\
& \lambda_{1}(D) \geqslant 1 / k^{2} \rho^{2}, \quad k>2 .
\end{aligned}
$$

In that paper Osserman suggests that one might be able to get a bound of the form $\lambda_{1}(D) \geqslant c / k \rho^{2}$. Taylor [6] proved that such a bound exists, although no explicit constant $c$ was given. Recently Cheng [2] has shown, using a completely different method, that $c$ can be taken to be $1 /(14000 \pi)^{2}$.

In this note we show

$$
\left.\lambda_{1}(D) \geqslant 1 / 2 k \rho^{2} \text { for } k \geqslant 2 \quad \text { (i.e. } c=\frac{1}{2}\right) \text {. }
$$

The method used here is similar to the one used in [4]. The method is useful only for plane domains, whereas Taylor's, Hayman's, and Cheng's methods are useful in higher dimensions or for variable curvatures.

TheOREM. Let $D$ be a connected, $k$-connected, domain in the plane with inradius $\rho$. Let $A$ represent the area of $D$ and $L$ represent the total boundary length. Then

$$
\begin{aligned}
L / A & \geqslant 1 / \rho, \quad k=1,2 . \\
L / A & \geqslant 2 /(1+\sqrt{k-1}) \rho \geqslant \sqrt{2} / \sqrt{k} \rho, \quad k \geqslant 2 . \\
\lambda_{1}(D) & \geqslant 1 / 4 \rho^{2}, \quad k=1,2 . \\
\lambda_{1}(D) & \geqslant 1 /(1+\sqrt{k-1})^{2} \rho^{2} \geqslant 1 / 2 k \rho^{2}, \quad k \geqslant 2 .
\end{aligned}
$$

Received by the editors March 24, 1980.

1980 Mathematics Subject Classification. Primary 52A40.

${ }^{1}$ Supported in part by NSF Grant MCS 76-01692. 
Proof. Inequalities (1) and (2) (applied to subdomains of $D$ ) imply inequalities (3) and (4) by Cheeger's result [1] as modified by Osserman [4]. Inequality (1) (and (3)) were proved in [4]. It is a simple computation to see $\sqrt{2} \sqrt{k}>(1+\sqrt{k-1})$. Hence we need only show that for $k>2, L / A>2 /(1+\sqrt{k-1}) \rho$.

We consider two cases.

Case 1. $A \leqslant(1+\sqrt{k-1})^{2} \pi \rho^{2}$.

By the isoperimetric inequality for plane domains we have $L^{2} / A>4 \pi$. Hence $L / A \geqslant 2 \sqrt{\pi} / \sqrt{A} \geqslant 2 /(1+\sqrt{k-1}) \rho$.

Case 2. $A>(1+\sqrt{k-1})^{2} \pi \rho^{2}$.

By a standard argument in the plane $[4$, p. 548] one has

$$
\frac{\rho L}{A} \geqslant 1-\frac{\pi(k-2) \rho^{2}}{A} \geqslant 1-\frac{(k-2)}{(1+\sqrt{k-1})^{2}}=\frac{2}{(1+\sqrt{k-1})} .
$$

This proves the theorem.

REMARKS. Inequalities (1) and (2) are sharp for $k=2$, with equality for a circular annulus. For $k=1$ inequality (1) is strict but it is also the best possible, as was noted by Santaló [5, p. 155], as one sees by considering long thin rectangles. For $k \geqslant 3$ inequality (2) is strict and not the best possible. In this case one could ask for the best constants $C(k)$ such that $L / A>C(k) / \sqrt{k} \rho$. The theorem gives $C(k)>2 \sqrt{k} /(1+\sqrt{k-1})$, thus asymptotically $C(k)>2$ (i.e. for every $\varepsilon>0$ and for sufficiently large $k, C(k)>2-\varepsilon)$. By considering large disks with triangularly packed points removed, one can see that asymptotically $C(k) \leqslant 2 \sqrt{2 \pi / 3 \sqrt{3}}$. Thus the theorem gives an estimate which is sharp for $k=2$ and close to the best asymptotically.

As for inequality (4), Osserman $\left[4\right.$, p. 552] gives examples where $\lambda_{1}(D)<$ $\pi^{2} / k \rho^{2}$; thus the inequality is not too far from the best possible.

\section{REFERENCES}

1. J. Cheeger, A lower bound for the smallest eigenvalue of the Laplacian, Problems in Analysis, A Symposium in Honor of S. Bochner, Princeton Univ. Press, Princeton, N. J., 1970, pp. 195-199.

2. S.-Y. Cheng, On the Hayman-Osserman-Taylor inequality (preprint).

3. W. Hayman, Some bounds for principal frequency, Applicable Anal. 7 (1978), 247-254.

4. R. Osserman, A note on Hayman's theorem on the bass note of a drum, Comment. Math. Helv. 52 (1977), 545-555.

5. L. A. Santaló, Sobre el circulo de radio maximo contenido en un recinto, Rev. Un. Mat. Argentina 10 (1945), 155-167.

6. M. Taylor, Estimate on the fundamental frequency of a drum, Duke Math. J. 46 (1979), 447-453.

Department of Mathematics, University of California, Berkeley, California 94720

Current address: Department of Mathematics, University of Pennsylvania, Philadelphia, Pennsylvania 19104 